\title{
Integrating Peridynamics with Material Point Method for Elastoplastic Material Modeling*
}

\author{
Yao Lyu ${ }^{1}$, Jinglu Zhang ${ }^{1}$, Jian Chang ${ }^{1}$, Shihui Guo ${ }^{2}$, and Jian Jun Zhang ${ }^{1}$ \\ 1 National Centre for Computer Animation, Bournemouth University, Dorset, UK \\ \{ylyu, zhangj, jchang, jzhang\}@bournemouth.ac.uk \\ 2 School of Software, Xiamen University, Xiamen, China \\ guoshihui@xmu.edu.cn
}

\begin{abstract}
We present a novel integral-based Material Point Method (MPM) using state based peridynamics structure for modeling elastoplastic material and fracture animation. Previous partial derivative based MPM studies face challenges of underlying instability issues of particle distribution and the complexity of modeling discontinuities. To alleviate these problems, we integrate the strain metric in the basic elastic constitutive model by using material point truss structure, which outweighs differential-based methods in both accuracy and stability. To model plasticity, we incorporate our constitutive model with deviatoric flow theory and a simple yield function. It is straightforward to handle the problem of cracking in our hybrid framework. Our method adopts two time integration ways to update crack interface and fracture inner parts, which overcome the unnecessary grid duplication. Our work can create a wide range of material phenomenon including elasticity, plasticity, and fracture. Our framework provides an attractive method for producing elastoplastic materials and fracture with visual realism and high stability.
\end{abstract}

Keywords: Material Point Method · Peridynamics · Elastoplastic modeling.

\section{Introduction}

Physically-based modeling of elastoplastic material has been an active research topic for many years in compute graphics, particularly for its appealing application in visual effects industry. Scenes involving elastoplastic deformation are very common, for example, clothes moving with wind, rubber toys bouncing on the floor, or plastic board damaged by high speed impact. In order to model such realistic behaviors under different circumstances, the robust simulation method needs to be capable of handling complex topological changes and various contact responses, such as collision and cohesion. To find the simulation method that can

\footnotetext{
* This work is supported by National Natural Science Foundation of China (61702433, 61661146002), the Fundamental Research Funds for the Central Universities, the China Scholarship Council and Bournemouth University
} 
naturally model elastoplastic material along with complex topological changes is the current focus of the field.

Meshless simulation methods are powerful in dealing with complicated topological changes since it does not require high quality mesh and efforts to overcome the issues from severe mesh distortion. The MPM [23][24] is an extension of the particle-in-cell (PIC) method. It combines the Eulerian Cartesian grids and Lagrangian material points for tracking mass, momentum and deformation on particles [20]. It can naturally process material point distribution and self-collisions. It also has been proved to be especially suitable for animating materials that undergo large deformations [10]. Despite its physical realism and geometrical convenience, the traditional MPM solver has several disadvantages: 1)Due to the governing equation based on spatial derivatives of displacements, the results are sensitive to the underlying particle distribution [6]. Also it has difficulty in solving singularity along discontinuities. 2)To observe boundary details, MPM has to maintain a fine resolution grid which brings high computational costs for particle-grid transfer throughout the whole simulation domain. While researchers have extensively studied refining regions of particular interest by using adaptive grid [4], the ability to simulate detailed discontinuities dynamics, such as crack propagation, is still limited.

Recently, peridynamics has gained its popularity in meshless simulation for discontinuous deformation. It was originally proposed by Silling [16] and has been adopted mainly for studying fracture dynamics due to its integral based constitutive model. Peridynamics is not suitable for handling large deformation due to the fact that initial bond connections are not in consistent with the configuration where topological changes are severe. Based on this fact, there are not a lot of mature models and experiments in continuum mechanics being adopted by peridynamics methods for animating elastoplastic material [28]. Furthermore, its point-based nature leads additional efforts to handle contacts.

Aiming at alleviating above problems, we propose an integral-based MPM framework with peridynamics structure for modeling elastoplastic material and fractures. We present three main contributions:

Elasticity We equip material point with virtual bonds and family points. The elastic energy density function is redefined in an integral way with this truss structure. Varied stiffness of elastic materials can be simulated with high realism and stability.

Plasticity The virtual bond structure makes our model trivial to model plastic behaviors. We use a novel method to extract plasticity from the deviatoric part of constitutive model and accumulate plastic increment permanently at particle-grid transfer step.

Fracture We handle crack definition and propagation through screening virtual bonds. The fracture criterion combines the deformation status of single material point and the grid cell. We update fracture surfaces and fracture inner parts in different time integration methods. Our method avoids the difficulty of duplicating grids and large computation cost brought by multiple particle-grid transfer process. 
After discussing related work in Section 2, we outline our method and explain integral-based governing equation in Section 3. Section 4 describes the constitutive model for modeling elastoplastic material in details. Crack definition and propagation are discussed in Section 5. Experimental examples for evaluating our method and discussion of results are give in Section 6. In Section 7 we conclude the method and explain our future work.

\section{Related Work}

Elastoplastic continuum modeling Terzopoulos and Fleischer [26][27] pioneered the plastic simulation methods in computer graphics. O'Brien and colleagues [14] incorporated finite element method (FEM) with multiplicative plasticity model and obtained realistic motion for a much wider range of materials. Later, Gerszewski et al. [5] adopted deformation gradient for animating elastic behaviors based on point method. Levin [11] rediscretized elasticity on a Eulerian grid, which is similar to MPM grid. Based on previous hybrid grid and particle modeling method, Stomakhin [21] incorporated energetically consistent invertible elasticity model into MPM for modeling snow varying phase effects. Recently, Chen [3] presented a novel elastoplastic constitutive model to handle brittle fracture and ductile fracture in peridynamics-based framework. At the same time, many researchers focused on developing the real time and haptic simulator. For example, Salsedo et al. [15] designed the HAPTEX system using dynamically variable spatial resolution to reduce the computational burden during rendering the fabrics, which was extended by Bottcher [1][2] by implementing separated computation threads for different simulation scales.

Material Point Method MPM is a hybrid grid-particle method using a regular Eulerian Cartesian grid to treat self-collision and fracture naturally, proposed by Sulsky [23][24]. Later, Stomakhin et al. [21] introduced MPM into computer graphics and obtained a variety of snow phenomenon. Jiang and his colleagues proved that MPM is a useful method for granular materials by animating sand [9]. Tampubolon extended the MPM to simulate multi-phase behaviors through using multiple grids, such as porous sand and water interactions [25]. A majority of elastoplastic MPM works for computer graphics [22][8] focus on resolving intensive collision scenarios on the surface or the curve with millions degrees of freedom. Unlike above studies, our method incorporates integral-based constitutive model to replace typical partial derivative based model. This helps us alleviate the instability and difficulty issues from particle distribution of arbitrary elastoplastic deformation. With virtual bonds, constitutive model can demonstrate detailed topological changes smaller than grid cell.

Peridynamics Silling proposed the peridynamics theory [16] as an efficient nonlocal continuum theory to uniformly solve problems involving both continuities and discontinuities [18][3][6]. Instead of using spatial differential formulations, 
peridynamics uses spatial integral equations as the governing equations. Its application sparked the engineering field, such as multiscale material modeling [19] and crack dynamics [17]. Later, Levine [12] introduced the peridynamics theory to computer graphics. He revisited brittle fracture studies by characterizing peridynamics as spring-mass systems. Currently, most research [3][6] focused on how to reformulate elastic constitutive models and produce persuasive effects. However, the theoretical equivalence of peridynamics compared to continuum mechanics remains unclear [28]. A lot of mature theories and experiments in continuum mechanics have not been adopted for peridynamics in computer graphic. This motivates us to define the integral-based constitutive model equipped with peridynamics structure within MPM framework for versatile elastoplastic deformation and self-collision detection.

\section{Method}

The governing equation of MPM arises from basic conservation of mass and momentum [21]. Back to weak formulation which is obtained by multiplying the balance of momentum and integrating the governing equation over initial volume, we propose an integral force density function $F^{s}\left(x_{p}\right)$ to replace spatial derivatives of displacement and redefine the weak formulation as:

$$
\int_{\Omega} \rho a_{p} \delta u_{p} d \Omega+\int_{\Omega} \rho F^{s}\left(x_{p}\right) \delta u_{p} d \Omega=\int_{\Omega} \rho b_{p} \delta u_{p} d \Omega+\int_{\Gamma_{\tau}} \rho \delta u_{p} \overline{\tau_{p}} d \Gamma
$$

where $\Omega$ denotes the integrating region in the current configuration, $\rho$ is density, $a_{p}$ is the acceleration of particle $\mathrm{p}, \delta u_{p}$ is the virtual displacement (infinitesimal feasible changes where constraints remain satisfied). $b_{p}$ is the body force, for example, gravity. $\tau_{p}$ is the surface traction on part of the boundary $\Gamma_{\tau}$.

$$
F^{s}\left(x_{p}\right)=\int_{H_{x_{p}}}\left[T<x_{p}, x_{p}{ }^{\prime}>-T<x_{p}{ }^{\prime}, x_{p}>\right] / \rho d H_{x_{p}}
$$

$F^{s}\left(x_{p}\right)$ is the internal force density function constructed in an integral way. $x_{p}{ }^{\prime}$ is the neighbor point of point $x_{p}$. When incorporating peridynamics theory, we construct the truss structure for each material point as: the neighbor material points $H_{x_{p}}$ of material point $x_{p}$ are referred as its family members. $x_{p}$ has interaction with all its family members at same time. Each interaction is operated by a virtual bond. $T<x_{p}, x_{p}^{\prime}>$ represents the interaction force between $x_{p}$ and $x_{p}{ }^{\prime}$. The $F^{s}\left(x_{p}\right)$ avoids using the spatial derivatives of displacements $\frac{\delta u_{x_{p}}}{\delta x_{p}}$. We will discuss details of the constitutive model in Section 4.

In the MPM framework, the material domain at $t^{n}$ is discretised with particles at $x_{p}^{n}$. Each particle has volume $V$, mass $m_{p}$, velocity $v_{p}^{n}$, and other physical quantities, such as deformation matrix $F_{p}$, Lamé parameters $\mu_{p}$ and $\lambda_{p}$, plastic yield parameters $\psi_{p}$. In each time step, a new grid is generated. Grid node I is used to store nodal parameters, such as position $x_{I}$, mass $m_{I}$, velocity $v_{I}$, force 
$f_{I}$. Our framework adopts dyadic products of one-dimensional cubic B-splines as basic weight function in [21] during particle-grid transfer process. Here we outline the full update procedure:

1)Particle-to-grid transfer Transfer material point mass $m_{p}$ and momentum $(m v)_{p}$ to the grid.

2)Compute internal forces The internal force of grid node I is calculated by the stress tensor of each point, presented as $f_{I}^{I N T}=\sum_{N p} m_{p} N_{I}\left(x_{p}\right) F^{s}\left(x_{p}\right) V$. Our method presents this equation based on our integral-based energy function. The detailed description of the update rule for $F^{s}\left(x_{p}\right)$ is given in Section 4.

3)Update Grid Momentum Nodal velocities are updated by $\widehat{v}_{i}^{n+1}=v_{i}^{n}+$ $\triangle t f_{I} / m_{i}^{n}$ for explicit time integration. $f_{I}$ is the total force.

4)Grid-based body collisions Grid velocity $v_{I}^{n+1}$ is updated by collision field and friction parameter from [21].

5)Grid-to-particle transfer Transfer updated nodal velocity $v_{I}^{n+1}$ and momentum $(m v)_{I}^{n+1}$ to particle.

6)Particle collisions Modify $v_{p}^{n+1}$ by collision field on particle level to obtain detailed deformation behaviors on the boundary.

7)Fracture Based on the current particle distribution and the deformation status of grid cell, we remove virtual bond that intersects with fracture plane. The fracture model is discussed in Section 5 .

\section{Constitutive Models}

In this section, we describe our constitutive model in details. We start with elastic model, then incorporate plasticity in a consistent frame.

\subsection{Elastic Model}

When we only consider elasticity in continuum mechanics, the elastic energy density function [20] can be defined as:

$$
E^{s}=\mu\left\|F_{e}-R_{e}\right\|^{2}+\frac{\lambda}{2}\left(J_{e}-1\right)^{2}
$$

where $F_{e}$ is the deformation gradient tensor, $R_{e}$ is rotation matrix and $J_{e}=$ $\operatorname{det}\left(F_{e}\right)$. We can decompose this equation into the combination of two parts in the view of different contributions to topological changes: $\mu\left\|F_{e}-R_{e}\right\|^{2}$ as deviatoric part; $\frac{\lambda}{2}\left(J_{e}-1\right)^{2}$ as isotropic part. However, $F_{e}$ is based on the spatial derivatives of displacement which leads to the inability of constitutive model to compute singularity issues, such as discontinuities. We adopt the concept of integral deformation matrix $\overline{F_{p}}$ in [6] to describe the local deformation which has similar meaning to $F_{e}$ but is represented by the integration of displacement and peridynamics structure. When the initial bond state and deformed bond between material point $x_{p}$ and $x_{p}{ }^{\prime}$ are $X=x_{p}-x_{p}{ }^{\prime}$ and $Y=y_{p}-y_{p}{ }^{\prime}$, deformation matrix $\overline{F_{p}}$ shown in Equation 4 represents the average deformation status of point $x_{p}$. $H_{x_{p}}$ represents all family members. $w(Y)$ is linear weight function. $\otimes$ is dyadic 
product operator defined by Silling [16]. Thus an average deformed bond is calculated as $\bar{Y}=\overline{F_{i}} X$.

$$
\overline{F_{p}}=\left[\sum_{H_{x_{p}}} w(Y) Y \otimes X\right]\left[\sum_{H_{x_{p}}} w(Y) \otimes X\right]^{-1}
$$

With these concepts, Equation 3 is reformulated as the combination of deviatoric component and isotropic component :

$$
E^{s}=\sum_{H_{x_{p}}} w(Y)\left(\mu E^{d e v}+\frac{\lambda}{2} E^{i s o}\right)
$$

$E^{\text {dev }}=\left(\frac{|\bar{Y}|}{|X|}-1\right)^{2}$ describes deformed energy similar to mass spring system but removes the influence from different bond length in order to simulate material with the same stiffness. It uses the average deformed bond length. Similarly, $E^{\text {iso }}=\left(\frac{|Y|}{|X|}-1\right)^{2}$ represents single bond deformation energy.

Then the elastic force density function $T<x_{p}, x_{p}{ }^{\prime}>$ for the material point pair of $x_{p}$ and $x_{p}{ }^{\prime}$ is obtained through $\frac{\partial \psi}{\partial y_{p}}$, as:

$$
T<x_{p}, x_{p}{ }^{\prime}>=\frac{2 \mu w}{|X|^{2}}(\bar{Y}-|X| \operatorname{dir} \bar{Y})+\frac{\lambda w}{|X|^{2}}(Y-|X| \operatorname{dir} Y)
$$

where $\frac{2 \mu w}{|X|^{2}}(|\bar{Y}|-|X|) \operatorname{dir} \bar{Y}$ depends on all neighbours, so it presents the shear

stress effects. $\frac{\lambda w}{|X|^{2}}(|Y|-|X|) \operatorname{dir} Y$ denotes spring force between by $x_{p}$ and $x_{p}{ }^{\prime}$, so the direction follows the deformed bond. Using Equation 2 and Equation 6, the internal force of grid node I for updating nodal momentum is:

$$
f_{I}^{I N T}=\sum m_{p} N_{I}\left(x_{p}\right)\left[\sum_{H_{x_{p}}} w(Y)\left(T<x_{p}, x_{p}{ }^{\prime}>-T<x_{p}{ }^{\prime}, x_{p}>\right) V\right]
$$

Compared to many existing methods, our method only needs the current virtual bond state $Y$, so the local step is fast. Our method avoids the singular value decomposition (SVD) to extract elastic deformation gradient in [21]. The advantages of avoiding SVD are obvious: we obtain a better stability for simulation with large time steps as in [6]; also reduce the complexity of plasticity definition in MPM framework.

\subsection{Plastic Model}

Many methods [21][29] take out the part of elastic deformation gradient tensor that exceeds the yield function and push it into plastic deformation gradient calculation. Due to the hybrid structure in our method, the plasticity can be extracted simply on our integral-based elastic model. Singularity issues can be overcome easily without any extra efforts.

Our plastic model is purely from deviatoric plastic flow [3] theory. Firstly, we reformulate our elastic model in Equation 6 in order to adapt it for modeling 
plasticity. $|\bar{Y}|-|X|$ and $|Y|-|X|$ are average and single bond extension. When the deformation is smooth enough under small neighbor horizon, we predict $|\bar{Y}|-|X| \approx|Y|-|X|$. Then we have:

$$
T<x_{p}, x_{p}{ }^{\prime}>=\frac{2 \mu w}{|X|^{2}}(|Y|-|X|) \operatorname{dir} \bar{Y}+\frac{\lambda w}{|X|^{2}}(|Y|-|X|) \operatorname{dir} Y
$$

Based on the plastic flow theory, the unified displacement is decomposed into isotropic and deviatoric part, $e=(|Y|-|X|) /|X|=e^{i s o}+e^{\text {dev }}$. Plastic deformation $e^{p}$ is extracted from $e^{d e v}$. Then we integrate the plasticity into Equation 8:

$$
T<x_{p}, x_{p}{ }^{\prime}>=\frac{2 \mu w}{|X|}\left(e^{i s o}+e^{d e v}-e^{p}\right) \operatorname{dir} \bar{Y}+\frac{\lambda w}{|X|}\left(e^{i s o}+e^{d e v}-e^{p}\right) \operatorname{dir} Y
$$

We make the best use of our bond-particle-grid structure and define the yield function as $f\left(E_{\text {dev }}\right)$ :

$$
E_{\text {dev }}=\left(\frac{2 \mu w}{|X|}+\frac{\lambda w}{|X|}\right)\left(e^{d e v}-e^{p}\right), f\left(E_{\text {dev }}\right)=\frac{\left(E_{\text {dev }}\right)^{2}}{2}-\psi_{p}
$$

where $\psi_{p}$ is controllable plastic material parameter. We use $f\left(E_{\text {dev }}\right)$ to decide if the current configuration enters the plastic regime. If $f\left(E_{\text {dev }}\right)<0$, the deformation is still within the elastic domain. If $f\left(E_{d e v}\right)>0$, part of deformation occurred as plasticity. We project the deformation back to yield surface and add plastic increment $\Delta e^{p}$ to $e^{p}$ permanently as Equation 11.

$$
\Delta e^{p}=\frac{|X|}{(\lambda)}\left[E_{d e v}-\sqrt{2 \psi_{p}} \operatorname{sign}\left(E_{d e v}\right)\right]
$$

This model is still valid for elastic when $e^{p}$ varnishes in the equations. This constitutive model can be used for both elastic and elastoplastic materials in MPM.

\section{$5 \quad$ Fracture}

Crack simulation is a bottleneck of the MPM [13]. To processing the discontinuities at the interfaces, special treatments for creating cracks and partitioning fracture fragments into multigrid and multiple velocity fields are approaches [7]. When an excessive number of cracking interfaces are involved, the computation of multiple grid transferring can be very expensive. Additionally, the strategy to duplicate grid is limited because during simulation small fragments are numerous and randomly generated so it is hard to duplicate grid for each crack interface.

The dynamics with discontinuities is straightforward to compute in our MPM framework. If we simply remove over-deformed bonds like [3], it leads to numerous small fragments in deformed area rather than several crack lines after 
collision happens. We now propose to generate crack cut by fracture plane based on analyses of single point and global deformation status in gird cell.

Firstly, we define the fracture criterion by removing the plastic displacement as:

$$
l=\frac{e-e^{p}}{|X|\left(1+p_{\text {inactive }}\right)}
$$

where $p_{\text {inactive }}$ is the percentage of broken bond in total bond numbers in the grid cell where the material point stays.

After screening the material points whose $l$ exceeds the threshold, we use cluster method to sort these points into several deformed areas based on position and normal. For each area, we calculate the central point and the largest deformed bond. Next step we use the central point position and the bond direction as normal to construct fracture plane for each area. Any bond intersected by the fracture plane will be removed. That is how crack line occurs. With $p_{\text {inactive }}$, this method can effectively reduce the number of small fracture pieces. Because if one grid has too many broken bonds, the active bonds in this grid cell are less possible to be removed.

In the MPM, grid cell size decides the resolution of whole simulation. This also works for crack dynamics. In experiments, we transfer particle velocity to three grid cells in any direction to get stable, smooth results. When two sides of crack line are within this range, they will share another fragment information through transfer. Therefore using only one grid leads to "fracture sticky to each other" effects. We alleviate this problem by applying two time integration ways: material points on the crack surface are updated by its own bond forces; other material points which don't have any broken bonds (in the fragment inner parts) are updated by grids as normal. Thus we avoid the information mixture of different crack fragments.

\section{Result and Discussion}

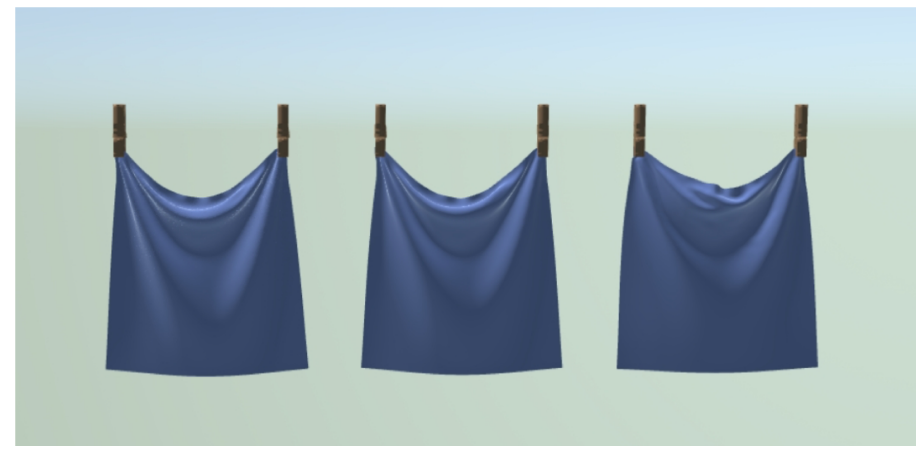

Fig. 1: The moving cloths with different material parameters show different bending stiffness. From left to right: $\mu=2 \times 10^{4}, \mu=1.5 \times 10^{5}, \mu=1 \times 10^{6}$. 

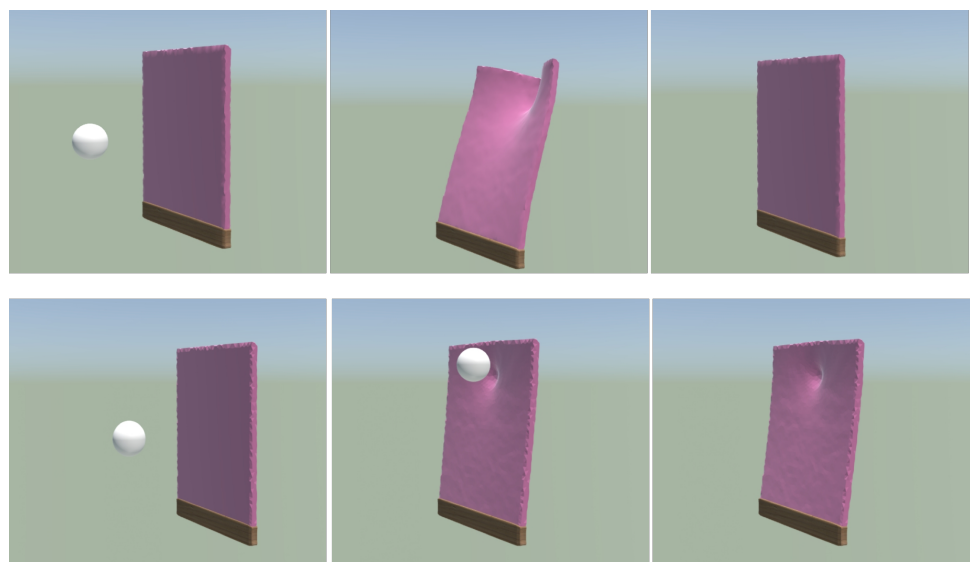

Fig. 2: Shooting ball to elastic and plastic boards show visibly different results. In the first row, elastic board can recover to initial shape after the collision. The second row shows that plastic board keeps deformed topology.

We implemented our method and tested examples in this section. All our examples are run in Houdini software, including material point discretization, vorinoi fracture generation and rendering. We use Houdini Development Kit(HDK) tools to customise nodes for material point dynamics. Eigen library is equipped with dynamics nodes. We use explicit time integration for the ease of implementation.

Implementation. We list the modeling types, parameter settings and the performance data in Table 1 for all the examples presented in the paper. We add ghost particles on object boundary to guarantee that each material point have similar family density.

Elastoplastic model validation. We use several examples with different material properties to evaluate our method. Fig. 1 shows the examples of garment anchored by clothespins. With varied bending stiffness parameters $\mu$, the experiments present the realistic and fine wrinkles. Fig. 2 shows the comparison of elastic board and plastic board collided by a ball. Our model can create correct behaviors. With complex topology objects, this method still works as in Fig. 3. Fig. 4 shows the simulation of ductile plastic fracture. Fig. 5 demonstrates the stretching beams deformation with different material stiffness.

Limitations and Future work. This paper presents a lot of examples with elastic and plastic material. However, there are still some works which need to be achieved in the future. Firstly, we represent fracture with Houdini voronoi structure which is represented by polygon with random vertex number. It is unable to generate the arbitrary fracture shape. The resolution is limited to the number and size of voronoi pieces. Incorporating tetrahedron structure for embedded geometry modeling is the future option for cooking detailed cracking interfaces. Secondly, we only apply plastic fracture models with deformed topology. Some important complex models should be covered in future work, such as brittle 

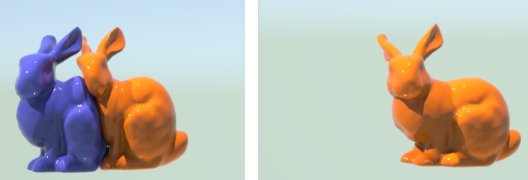

(a)

(b)
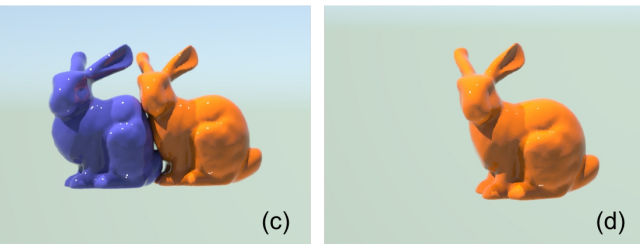

Fig. 3: The collision between two identical bunnies with different materials. This example demonstrates the different deformation of elastic bunny (in the first row) and plastic bunny (in the second row). Collision happens in (a) and (c). Elastic bunnies can recover as in (b). Plastic bunny deforms afterwards in (d).

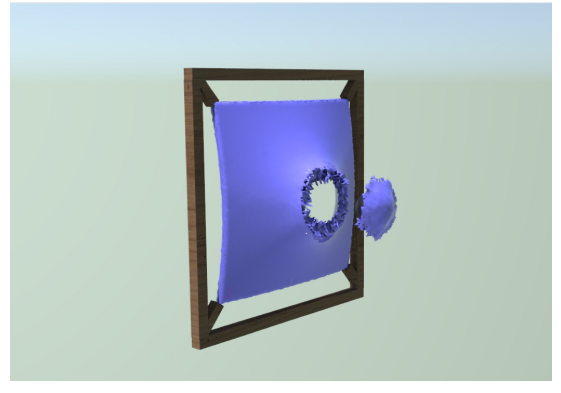

Fig. 4: Simulation of plastic wall collided by a sphere.

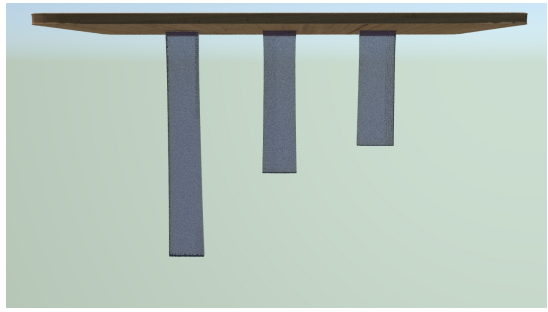

Fig. 5: Stretch beams with different material parameters. From left to right, bending stiffness $\mu$ are: $0,50,500$.

Table 1: modeling information for all examples

\begin{tabular}{|c|c|c|c|c|c|c|c|}
\hline & Type & $\begin{array}{c}\text { Grid cell } \\
\text { Size }(\mathrm{cm})\end{array}$ & $\lambda(\mathrm{Kpa})$ & $\mu(\mathrm{Kpa})$ & $\Psi_{\mathrm{p}}$ & $\begin{array}{c}\text { Fracture } \\
\text { threshold }\end{array}$ & $\Delta \mathrm{t}(\mathrm{ms})$ \\
\hline Cloth & Mesh & 0.005 & $1 \times 10^{6}$ & $2 \times 10^{4}, 1.5 \times 10^{5}, 1 \times 10^{6}$ & $1 \times 10^{25}$ & $1 \times 10^{10}$ & 0.1 \\
\hline Elastic board & Mesh & 0.02 & $3 \times 10^{6}$ & $1.5 \times 10^{5}$ & $1 \times 10^{25}$ & $1 \times 10^{15}$ & 0.02 \\
\hline Plastic board & Mesh & 0.02 & $5 \times 10^{6}$ & $1.5 \times 10^{5}$ & 1000 & $1 \times 10^{15}$ & 0.0001 \\
\hline Elastic bunny & Particles & 0.01 & $1 \times 10^{5}$ & $1 \times 10^{5}$ & $1 \times 10^{25}$ & $1 \times 10^{25}$ & 0.1 \\
\hline Plastic bunny & Particles & 0.01 & $1 \times 10^{6}$ & $3 \times 10^{5}$ & 300 & $1 \times 10^{25}$ & 0.01 \\
\hline Broken board & Voronoi & 0.005 & $1 \times 10^{6}$ & $5 \times 10^{4}$ & 500 & 0.05 & 0.001 \\
\hline Stretching beam & Mesh & 0.05 & 500 & $0,50,500$ & $1 \times 10^{25}$ & $1 \times 10^{25}$ & 100 \\
\hline
\end{tabular}


glass crack and complex crack propagation. Finally, we use explicit integration for its straightforwardness in HDK. It requires very small substeps for dealing with huge displacement. Alternatively, the current study implements projective dynamics implicit integration method for fast simulation. It can obtain stable and robust results under large substeps. Our future work will focus on addressing above limitations and obtaining versatile and realistic elastoplastic performance.

\section{Conclusion}

We present an integral-based constitutive model within the Material Point Method framework. Our method demonstrates various elastic deformation and plastic deformation scenarios. Fracture can be modelled robustly without any singularity issues. Additionally, our method presents a novel integral-based view for multimaterial modeling and fractures modeling, which has potential to inspire future research in the field.

\section{References}

1. Böttcher, G.: Haptic Interaction with Deformable Objects: Modelling VR Systems for Textiles. Springer Science \& Business Media (2011)

2. Bottcher, G., Allerkamp, D., Wolter, F.E.: Virtual reality systems modelling haptic two-finger contact with deformable physical surfaces. In: 2007 International Conference on Cyberworlds (CW'07). pp. 292-299. IEEE (2007)

3. Chen, W., Zhu, F., Zhao, J., Li, S., Wang, G.: Peridynamics-based fracture animation for elastoplastic solids. In: Computer Graphics Forum. vol. 37, pp. 112-124. Wiley Online Library (2018)

4. Gao, M., Tampubolon, A.P., Jiang, C., Sifakis, E.: An adaptive generalized interpolation material point method for simulating elastoplastic materials. ACM Transactions on Graphics (TOG) 36(6), 223 (2017)

5. Gerszewski, D., Bhattacharya, H., Bargteil, A.W.: A point-based method for animating elastoplastic solids. In: Proceedings of the 2009 ACM SIGGRAPH/Eurographics Symposium on Computer Animation. pp. 133-138. ACM (2009)

6. He, X., Wang, H., Wu, E.: Projective peridynamics for modeling versatile elastoplastic materials. IEEE transactions on visualization and computer graphics 24(9), 2589-2599 (2018)

7. Homel, M.A., Herbold, E.B.: Field-gradient partitioning for fracture and frictional contact in the material point method. International Journal for Numerical Methods in Engineering 109(7), 1013-1044 (2017)

8. Jiang, C., Gast, T., Teran, J.: Anisotropic elastoplasticity for cloth, knit and hair frictional contact. ACM Transactions on Graphics (TOG) 36(4), 152 (2017)

9. Jiang, C., Schroeder, C., Selle, A., Teran, J., Stomakhin, A.: The affine particlein-cell method. ACM Transactions on Graphics (TOG) 34(4), 51 (2015)

10. Jiang, C., Schroeder, C., Teran, J., Stomakhin, A., Selle, A.: The material point method for simulating continuum materials. In: ACM SIGGRAPH 2016 Courses. p. 24. ACM (2016)

11. Levin, D.I., Litven, J., Jones, G.L., Sueda, S., Pai, D.K.: Eulerian solid simulation with contact. ACM Transactions on Graphics (TOG) 30(4), 36 (2011) 
12. Levine, J.A., Bargteil, A.W., Corsi, C., Tessendorf, J., Geist, R.: A peridynamic perspective on spring-mass fracture. In: Proceedings of the ACM SIGGRAPH/Eurographics Symposium on Computer Animation. pp. 47-55. Eurographics Association (2014)

13. Liang, Y., Benedek, T., Zhang, X., Liu, Y.: Material point method with enriched shape function for crack problems. Computer Methods in Applied Mechanics and Engineering 322, 541-562 (2017)

14. O'brien, J.F., Bargteil, A.W., Hodgins, J.K.: Graphical modeling and animation of ductile fracture. In: ACM transactions on graphics (TOG). vol. 21, pp. 291-294. ACM (2002)

15. Salsedo, F., Fontana, M., Tarri, F., Ruffaldi, E., Bergamasco, M., MagnenatThalmann, N., Volino, P., Bonanni, U., Brady, A., Summers, I., et al.: Architectural design of the haptex system. In: submitted to the Proceedings of this Conference (2005)

16. Silling, S.A.: Reformulation of elasticity theory for discontinuities and long-range forces. Journal of the Mechanics and Physics of Solids 48(1), 175-209 (2000)

17. Silling, S.A., Askari, A.: Peridynamic model for fatigue cracking. SAND2014-18590. Albuquerque: Sandia National Laboratories (2014)

18. Silling, S.A., Epton, M., Weckner, O., Xu, J., Askari, E.: Peridynamic states and constitutive modeling. Journal of Elasticity 88(2), 151-184 (2007)

19. Silling, S.A., Askari, A.: Practical peridynamics. Tech. rep., Sandia National Lab.(SNL-NM), Albuquerque, NM (United States) (2014)

20. Stomakhin, A., Howes, R., Schroeder, C., Teran, J.M.: Energetically consistent invertible elasticity. In: Proceedings of the ACM SIGGRAPH/Eurographics Symposium on Computer Animation. pp. 25-32. Eurographics Association (2012)

21. Stomakhin, A., Schroeder, C., Chai, L., Teran, J., Selle, A.: A material point method for snow simulation. ACM Transactions on Graphics (TOG) 32(4), 102 (2013)

22. Stomakhin, A., Teran, J., Selle, A.: Augmented material point method for simulating phase changes and varied materials (Jul 2 2015), uS Patent App. 14/323,798

23. Sulsky, D., Chen, Z., Schreyer, H.L.: A particle method for history-dependent materials. Computer methods in applied mechanics and engineering 118(1-2), 179-196 (1994)

24. Sulsky, D., Zhou, S.J., Schreyer, H.L.: Application of a particle-in-cell method to solid mechanics. Computer physics communications 87(1-2), 236-252 (1995)

25. Tampubolon, A.P., Gast, T., Klár, G., Fu, C., Teran, J., Jiang, C., Museth, K.: Multi-species simulation of porous sand and water mixtures. ACM Transactions on Graphics (TOG) 36(4), 105 (2017)

26. Terzopoulos, D., Fleischer, K.: Modeling inelastic deformation: viscolelasticity, plasticity, fracture. In: ACM Siggraph Computer Graphics. vol. 22, pp. 269-278. ACM (1988)

27. Terzopoulos, D., Platt, J., Barr, A., Fleischer, K.: Elastically deformable models. ACM Siggraph Computer Graphics 21(4), 205-214 (1987)

28. Xu, L., He, X., Chen, W., Li, S., Wang, G.: Reformulating hyperelastic materials with peridynamic modeling. In: Computer Graphics Forum. vol. 37, pp. 121-130. Wiley Online Library (2018)

29. Zhu, B., Lee, M., Quigley, E., Fedkiw, R.: Codimensional non-newtonian fluids. ACM Transactions on Graphics (TOG) 34(4), 115 (2015) 